\title{
An integrative simulation model linking major biochemical reactions of actin-polymerization to structural properties of actin filaments
}

\author{
Aliaksandr A. Halavatyi ${ }^{\mathrm{a}, \mathrm{b}, 1}$, Petr V. Nazarov ${ }^{\mathrm{a}, \mathrm{c}, 1}$, Sandrine Medves ${ }^{\mathrm{b}}$, Marleen van Troys ${ }^{\mathrm{d}, \mathrm{e}}$, \\ Christophe Ampe ${ }^{\mathrm{d}, \mathrm{e}}$, Mikalai Yatskou ${ }^{\mathrm{b}, \mathrm{c}}$, Evelyne Friederich ${ }^{\mathrm{b}, \mathrm{c}, *}$ \\ a Department of Systems Analysis, Belarusian State University, 4, Nezavisimosti Ave, 220030 Minsk, Belarus \\ ${ }^{\mathrm{b}}$ Faculty of Sciences, Technology and Communication, Life Sciences Research Unit, University of Luxembourg, 162 A, avenue de la Faïencerie, L-1511 Luxembourg, Luxembourg \\ c Centre de Recherche Public Santé, 84, Rue Val Fleuri, L-1526 Luxembourg, Luxembourg \\ d VIB Department for Molecular Biomedical Research, UGent, VIB, A. Baertsoenkaai 3, Gent, Belgium \\ e Department of Biochemistry, Faculty of Medicine and Health Sciences, UGent, A. Baertsoenkaai 3, Gent, Belgium
}

\section{A R T I C L E I N F O}

\section{Article history:}

Received 16 September 2008

Received in revised form 10 November 2008

Accepted 11 November 2008

Available online 27 November 2008

\section{Keywords:}

Actin

Filament

Polymerization

Model

Monte Carlo simulation

Software

\begin{abstract}
A B S T R A C T
We report on an advanced universal Monte Carlo simulation model of actin polymerization processes offering a broad application panel. The model integrates major actin-related reactions, such as assembly of actin nuclei, association/dissociation of monomers to filament ends, ATP-hydrolysis via ADP-Pi formation and ADP-ATP exchange, filament branching, fragmentation and annealing or the effects of regulatory proteins. Importantly, these reactions are linked to information on the nucleotide state of actin subunits in filaments (ATP hydrolysis) and the distribution of actin filament lengths. The developed stochastic simulation modelling schemes were validated on: i) synthetic theoretical data generated by a deterministic model and ii) sets of our and published experimental data obtained from fluorescence pyrene-actin experiments. Build on an open-architecture principle, the designed model can be extended for predictive evaluation of the activities of other actin-interacting proteins and can be applied for the analysis of experimental pyrene actinbased or fluorescence microscopy data. We provide a user-friendly, free software package ActinSimChem that integrates the implemented simulation algorithms and that is made available to the scientific community for modelling in silico any specific actin-polymerization system.
\end{abstract}

(C) 2008 Elsevier B.V. All rights reserved.
Abbreviations: F-actin, Filamentous actin; nSRF model, non-structurally-resolved filament model; SRF model, structurally-resolved filament model; Pi, Phosphate; ATM, Globular actin (monomeric form) with incorporated ATP; ADM, Globular actin (monomeric form) with incorporated ADP; ATF, Filamentous actin protomer (F-actin) with incorporated ATP; APF, Filamentous actin protomer (F-actin) with incorporated ADP-Pi; ADF, Filamentous actin protomer (F-actin) with incorporated ADP; FTB, Barbed ends of filaments, terminating by ATP-actin; FPB, Barbed ends of filaments, terminating by ADP-Pi-actin; FDB, Barbed ends of filaments, terminating by ADP-actin; FTP, Pointed ends of filaments, terminating by ATP-actin; FPP, Pointed ends of filaments, terminating by ADP-Pi-actin; FDP, Pointed ends of filaments, terminating by ADP-actin; CBM, Barbed-end capping protein (capper) in monomer (free) form; CBF, Barbed-end capper bound to filament; CPM, Pointed-end capper in monomer (free) form; CPF, Pointed-end capper bound to filament; FOM, Formin in monomer (free) form; FOF, Formin, bound to filament barbed ends; ARM, Arp $2 / 3$ complex in monomer (free) form; ARF, Arp2/3 complex associated with filament; FRP, Arp2/3 complex associated with filament pointed end (pointed ends of filaments, terminating by ARP $2 / 3$ complexes); FRB, Arp $2 / 3$ complex associated with filament with no bound actins (barbed ends of filaments, terminating by ARP2/3 complexes).

* Corresponding author. Office address: Campus Limpertsberg, $162 \mathrm{~A}$, avenue de la Faïencerie, L-1511, Luxembourg. Tel.: +352 4666446645 .

E-mail address: evelyne.friederich@uni.lu (E. Friederich).

1 These authors contributed equally to this work.

\section{Introduction}

Actin is a protein capable of self-assembling into dynamic filaments that form the actin cytoskeleton. This process is required for many crucial physiological functions like morphogenesis, cell migration and division or cell communication. In cells, actin monomer assembly and filament organization are controlled in time and space by associated proteins [1-4]. Because of its essential functions in cells, it is not surprising that perturbations of the actin cytoskeleton are associated with numerous diseases, including cancer, myopathies- and neurodegenerative disorders. For example, uncontrolled migration of cancer cells leads to metastasis and is significantly dependent on perturbed actin polymerization $[5,6]$. In addition, several pathogens, like Listeria monocytogenes, use actin polymerization for their propulsion inside infected cells $[7,8]$. Thus, a thorough understanding of how actin polymerization is regulated to generate forces and movement will lead to a better understanding of how it contributes to these physiopathological processes [9].

Many biophysical models have been proposed for the mechanisms by which actin filament assembly generates force that is translated into movement (see [10] for review). In addition, specific biochemical 
models were established to evaluate the effects of regulatory proteins on the actin polymerization reaction such as nucleation and filament elongation in the presence of formins [11-13] or the Arp2/3 complex [14-16], effects of capping [14,17,18], or of severing proteins [19-21]. These deterministic models rely on solving differential equations. One of the efficient non-analytical approaches which in principle can describe the actin polymerization processes is the Monte Carlo simulation [22]. Obviously, stochastic Monte Carlo models have a considerable potential because: i) they can describe adequately many actin-polymerization processes simultaneously, such as spontaneous or stimulated actin nucleation, (un)branching, fragmentation, annealing, complete depolymerisation, (un)capping, two-step ATP-hydrolysis $[18,23]$; ii) in addition stochastic models have the advantage that they can be applied to complex situations involving structural information and distribution of actin filament lengths [24,25]. Moreover, Monte Carlo models are easy to understand, since the simulation algorithms are made from so-called first principles known on the studied systems, and not requiring a strong mathematical background $[26,27]$. However, so far, no comprehensive universal Monte Carlo model and software of actin-polymerization reactions with a broad application panel for various actin polymerization processes are available.

In this paper, we developed a systematic Monte Carlo simulation formalism and an computational tool for modelling and analysing the main actin polymerization reactions, uniquely including the nucleotide compositions of monomers and filaments, and the distribution of actin filament lengths. This tool was evaluated using well-characterized reactions such as actin association to and dissociation from filaments, assembly of actin nuclei (spontaneous and stimulated by capping-proteins or formin), filament capping-related reactions, ATPhydrolysis and ATP recharge of actin monomers, filament branching, fragmentation and annealing. The simulation models and computational algorithms were integrated in the stand-alone executable software package ActinSimChem.

\section{Model}

\subsection{The model formalism}

Actin polymerization is used by cells as a source of mechanical forces, which can be translated into cell propulsion. The process of actin assembly is influenced by the concentration of monomeric actin charged with ATP or ADP nucleotide, physical and biochemical conditions and, importantly, by different actin-regulatory proteins: actin nucleators such as the Arp 2/3 complex and formin, different end cappers and severing proteins like cofilins [9]. In this work, we considered the reactions between ATP- and ADP-actin monomers (ATM, ADM, $c f$. the section Abbreviations), formin (FOM, a protein

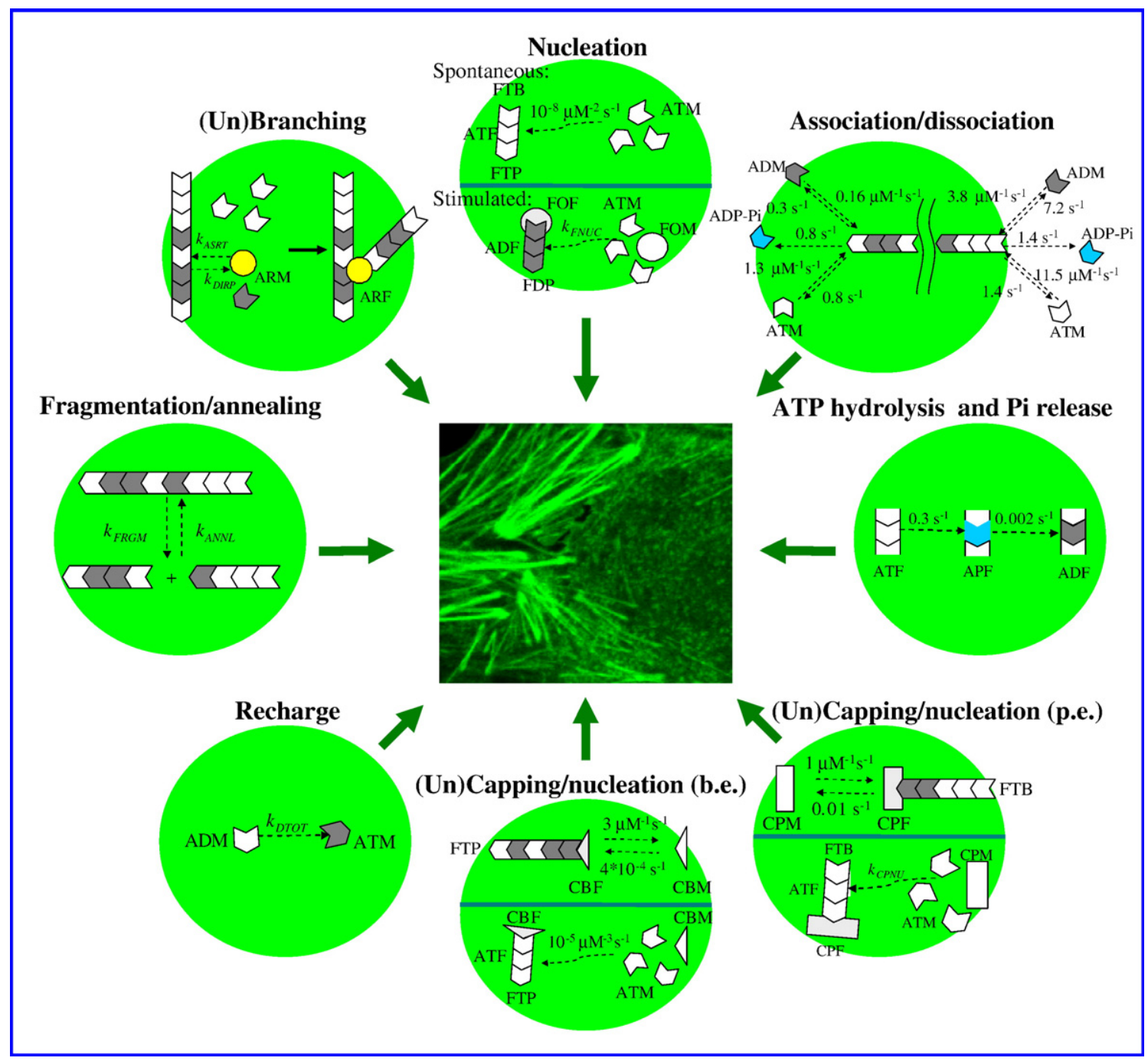

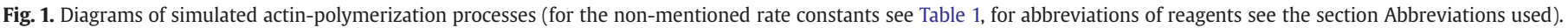


stimulating actin monomer assembly), actin nucleator Arp 2/3 complex (ARM), and end cappers (CBM, CPM, regulating actin filament dynamics at barbed (B) or pointed (P) ends). Kinetic actin polymerization experiments have shown that capping proteins also nucleate actin polymerization in the presence of excess actin ATPmonomers [28].

Diagrams, showing the formalization of actin polymerization processes, are given in Fig. 1. Actin monomers self-assemble to form trimers with the rate constant $k_{S N U C}$. Actin filaments grow from these "nuclei" by addition of monomers. The structural asymmetry of the filament ends is correlated with dissimilar assembly and disassembly rates. An actin filament grows under appropriate conditions approximately ten times faster at the barbed end than at its pointed end. The growing process is reversible and it is controlled by a polymerizationdepolymerization equilibrium. The aging of F-actins occurs when ATP hydrolyses with the rate constants $k_{\text {TTOP }}$ and $k_{P T O D}$ via the ADP-Pi "intermediate" state, consisting in ADP with a weakly associated phosphate. ATP hydrolysis results in weakening of actin subunit interaction and destabilization of the actin filament. Free ADP-actin monomers exchange their nucleotide for ATP because of the higher affinity of monomeric actin for ATP [29]; we introduced here the rate constant $k_{D T O T}$ to describe this process.
Free ends of a filament can be reversibly capped by either barbed or pointed end cappers. Capping proteins or formin promote actin nucleation, mostly by stabilizing actin trimers, at much higher rates ( $k_{C B N U}, k_{C P N U}$ and $k_{F N U C}$ ) when compared to the rate of spontaneous actin nucleation $\left(k_{S N U C}\right)$. In addition, formin being attached to the barbed end of nucleated actin oligomers, can significantly speed up barbed end elongation of the filament [11]. Branching reactions of Arp2/3 complex with F-actins are realized according to the mechanism of side branching [14]. The reactions of filament fragmentation and annealing were integrated as reported in [25]. We assume spontaneous fragmentation occurs in a manner invariant to position and nucleotide state of the actin-protomer (the rate constant $k_{F R G M}$ ). Annealing reaction consists in adhesion of free barbed ends and pointed ends of filaments with the rate constant $k_{A N N L}$. Other hypotheses on mechanisms of filament branching, fragmentation, annealing, nucleation, capping, can accordingly also be integrated within the frame of our simulation scheme.

To build the simulation model for actin polymerization we made the following assumptions:

- Actin-polymerization is simulated in a cubic volume of the size $V$ with periodic boundary conditions.
A

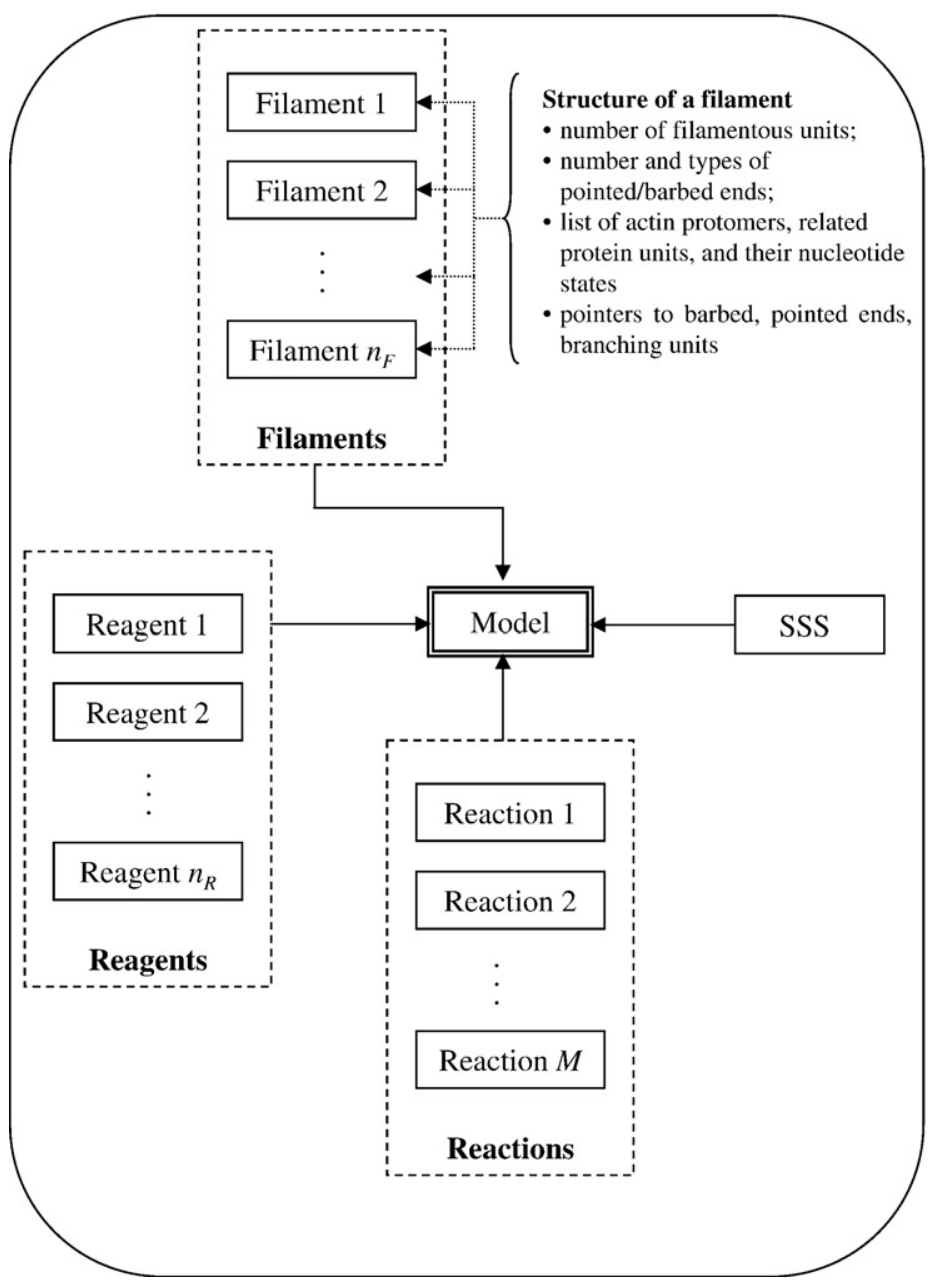

B

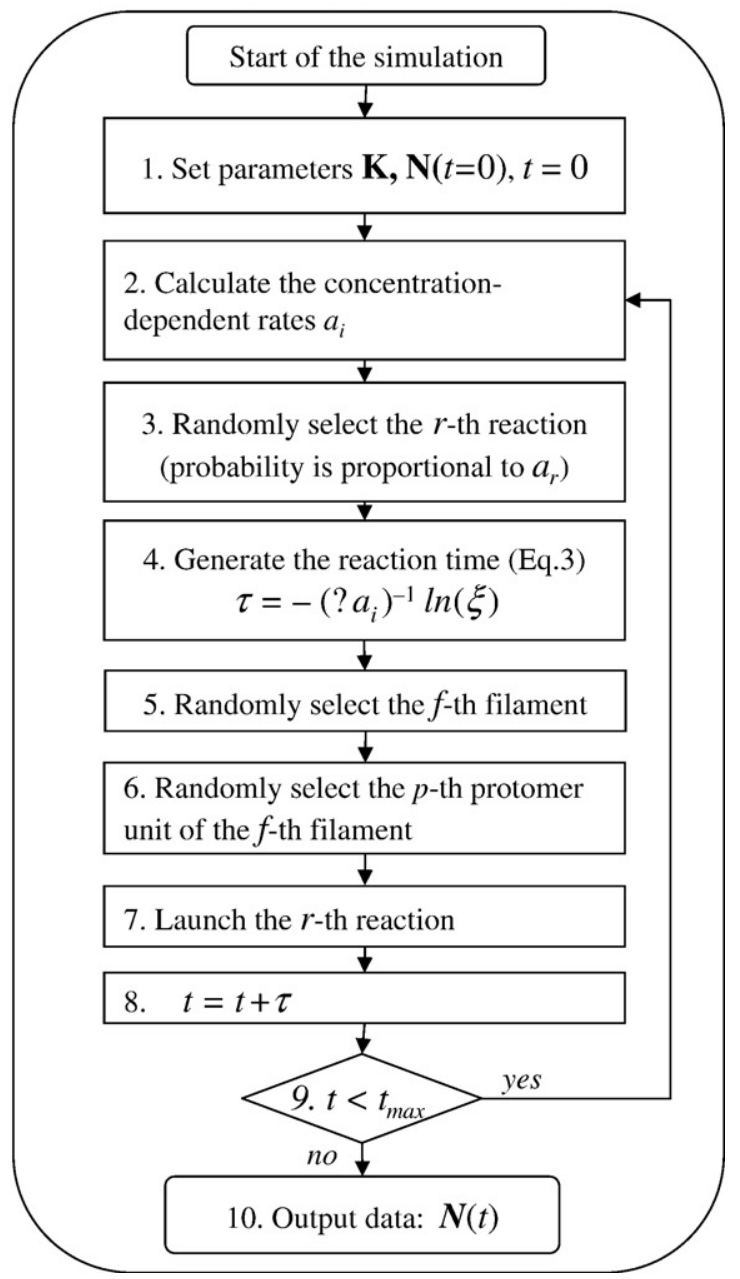

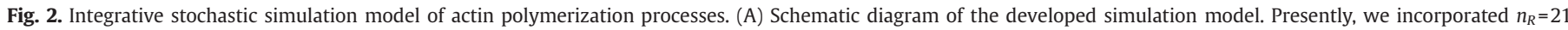

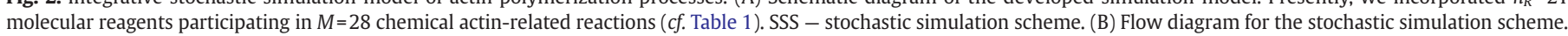
Vector $\mathbf{N}=\left\{N_{k}\right\}=\left(N_{A T M}, N_{A D M}, \ldots, N_{F R B}\right)$ contains the number of molecules of each reagent. The evolution vector $\mathbf{N}$ in time is denoted as $\mathbf{N}(t)$. 
- Since free actin monomers and free actin-associated proteins (cappers, Arp2/3, formins) diffuse rapidly [30-33], we assumed their spatial distribution is homogeneous.

- Quantities of monomeric proteins, i.e. the numbers of molecules $N_{A T M}, N_{C B M}$, etc. in the volume $V$, are calculated from the initial molecular concentrations $[\mathrm{ATM}]_{\mathrm{t}=0},[\mathrm{CBM}]_{\mathrm{t}=0}$, etc.

- Filaments are simulated as independent objects.

- The total concentrations of the monomeric proteins are constant over the volume $V$.

The flow diagram of the developed integrative stochastic simulation model of actin polymerization processes is shown in Fig. 2A. The core of this model is the ability to reproduce any complex actin polymerization system in a unique and universal way, whatever particular actin-based system is used. It combines various molecular reagents, chemical reactions and structures of actin filaments within a unique stochastic simulation scheme (Fig. 2B). The integrity of this modelling approach is based on an open-architecture principle, allowing easy incorporation of new reagents, additional actin-polymerization reactions, filament structures or even new stochastic simulation algorithms.

Presently, to simulate actin polymerization processes, we used $n_{R}=21$ molecular reagents participating in $M=28$ chemical actin-related reactions ( $c f$. Table 1 ). Reagents are globular and filamentous actins (with incorporated ATP/ADP-Pi/ADP nucleotide), barbed and pointed ends of filaments (terminated by ATP-/ADP-Pi-/ADP-actins), barbed and pointed end capping proteins, formin and the Arp $2 / 3$ complex (either as free forms or associated with filaments). The filaments are represented as consequences of protomer associations characterised by the number of protomers, the nucleotide states of these protomers, the number and types of pointed and barbed ends, pointers to filamentous ends and actin related proteins, branching units, i.e. the sites for growth of 'daughter' branches. Any 'daughter' branches to a 'mother' filament are similarly organised.

\subsection{The simulation of reactions}

To simulate the chemical interactions between molecular reagents in the actin filaments a proper discrete-event Monte Carlo simulation methodology is needed. Several stochastic simulation strategies have been implemented and compared. More specifically, we developed new simulation schemes based on the updates of the Gillespie's "direct" method and its modifications: the "first reaction" method [34], the "next reaction" method of Gibson-Bruck [35] and the $\tau$-leap algorithm [36]. The best results were obtained for the one generated on the base of the Gillespie's "direct" algorithm [see Appendix A]. A flow diagram of the stochastic simulation scheme implemented in our model is shown in Fig. 2B.

The simulation starts in block 1 by setting the volume $V$, the initial numbers of molecules $\mathbf{N}=\left(N_{A T M}, N_{A D M}, \ldots, N_{F R B}\right)$, and the rate constants $\mathbf{K}=\left(k_{S N U C}, k_{C B N U}, \ldots, k_{D T O T}\right)$. In block 2 the concentration-dependent reaction rates $a_{i}$, where $i$ is the index running for 28 reactions \{SNUC, $C B N U, \ldots, D T O T\}$ (cf. Table 1), are calculated using Eq. (1) or Eq. (2).

$a_{i}=k_{i}\left(10^{-6} N_{A} V\right)^{1-n_{i}} \prod_{j=1}^{n_{i}} N_{j}$,

where

$n_{i}$ is the number of reagents participating in the $i$-th reaction;

$k_{i}$ is the experimental concentration-independent rate constant, given in the same units as in Table $1\left(\mathrm{~s}^{-1}, \mu \mathrm{M}^{-1} \mathrm{~s}^{-1}, \mu \mathrm{M}^{-2} \mathrm{~s}^{-1}\right.$, and $\mu \mathrm{M}^{-3} \mathrm{~s}^{-1}$ for $n=1,2,3$, and 4 respectively);

$N_{A}$ is the number of Avogadro;

$N_{j}$ is the number of molecules of $j$-th type, contained in volume $V$. This number is linked with the concentration of the $j$-th reagent as $C_{j}=N_{j} / V$.
Table 1

Simulated reactions and corresponding rate constants

\begin{tabular}{|c|c|c|}
\hline Reaction and equation & Symbol & Values [Ref.] \\
\hline $\begin{array}{l}\text { Spontaneous nucleation of } \\
\text { the filament } \\
3 \mathrm{ATM} \rightarrow 3 \mathrm{ATF}+\mathrm{FTB}+\mathrm{FTP}^{*}\end{array}$ & $k_{S N U C}$ & $\begin{array}{l}2.3 \times 10^{-11} \mu \mathrm{M}^{-2} \mathrm{~s}^{-1}[51] \\
1.1 \times 10^{-9} \mu \mathrm{M}^{-2} \mathrm{~s}^{-1} \\
{[14] ; \sim 2 \times 10^{-8} \mu \mathrm{M}^{-2} \mathrm{~s}^{-1}[52]}\end{array}$ \\
\hline $\begin{array}{l}\text { Formin-initiated nucleation } \\
\text { FOM }+3 \text { ATM } \rightarrow 3 \text { ADF }+ \text { FOF }+ \text { FDP }\end{array}$ & $k_{F N U C}$ & $7 \times 10^{-5} \mu \mathrm{M}^{-3} \mathrm{~s}^{-1}[13]$ \\
\hline $\begin{array}{l}\text { Nucleation by barbed-end capping } \\
\text { protein } \\
\mathrm{CBM}+3 \mathrm{ATM} \rightarrow 3 \mathrm{ATF}+\mathrm{CBF}+\mathrm{FTP}\end{array}$ & $k_{C B N U}$ & $\begin{array}{l}\text { Used in simulations }-10^{-5} \mu \mathrm{M}^{-3} \mathrm{~s}^{-1} \text {; } \\
\text { for six ATM }-2.94 \times 10^{-5} \mu \mathrm{M}^{-6} \mathrm{~s}^{-1}[14]\end{array}$ \\
\hline $\begin{array}{l}\text { Nucleation by pointed-end capping } \\
\text { protein } \\
\mathrm{CPM}+3 \mathrm{ATM} \rightarrow 3 \mathrm{ATF}+\mathrm{CPF}+\mathrm{FTB}\end{array}$ & $k_{C P N U}$ & No data \\
\hline $\begin{array}{l}\text { ATP-actin association at barbed end } \\
\text { FxB**+ATM } \rightarrow \text { FTB }+ \text { ATF }\end{array}$ & $k_{A S T B}$ & $11.5 \mu \mathrm{M}^{-1} \mathrm{~s}^{-1}[53]$ \\
\hline $\begin{array}{l}\text { ADP-actin association at barbed end } \\
\text { FxB }+ \text { ADM } \rightarrow \text { FDB }+ \text { ADF }\end{array}$ & $k_{A S D B}$ & $3.8 \mu \mathrm{M}^{-1} \mathrm{~s}^{-1}[53]$ \\
\hline $\begin{array}{l}\text { ATP-actin association at pointed end } \\
\text { FxP }+\mathrm{ATM} \rightarrow \mathrm{FTP}+\mathrm{ATF}\end{array}$ & $k_{\text {ASTP }}$ & $1.3 \mu \mathrm{M}^{-1} \mathrm{~s}^{-1}[53]$ \\
\hline $\begin{array}{l}\text { ADP-actin association at pointed end } \\
\text { FxP }+ \text { ADM } \rightarrow \text { FDP }+ \text { ADF }\end{array}$ & $k_{A S D P}$ & $0.16 \mu \mathrm{M}^{-1} \mathrm{~s}^{-1}[53]$ \\
\hline $\begin{array}{l}\text { Dissociation of ATP-actin from } \\
\text { barbed end } \\
\text { FTB } \rightarrow \text { FxB + ATM(+FRP) }\end{array}$ & $k_{\text {DITB }}$ & $1.4 \mathrm{~s}^{-1}[53]$ \\
\hline $\begin{array}{l}\text { Dissociation of ADP-Pi-actin from } \\
\text { barbed end }\end{array}$ & $k_{D I P B}$ & $1.4 \mathrm{~s}^{-1}[25,53]$ \\
\hline $\mathrm{FPB} \rightarrow \mathrm{FxB}+\mathrm{ADM}(+\mathrm{FRP})$ & & \\
\hline $\begin{array}{l}\text { Dissociation of ADP-actin from } \\
\text { barbed end } \\
\text { FDB } \rightarrow \mathrm{FxB}+\mathrm{ADM}(+\mathrm{FRP})\end{array}$ & $k_{D I D B}$ & $7.2 \mathrm{~s}^{-1}[53]$ \\
\hline $\begin{array}{l}\text { Dissociation of ATP-actin from } \\
\text { pointed end }\end{array}$ & $k_{\text {DITP }}$ & $0.8 \mathrm{~s}^{-1}[53]$ \\
\hline $\mathrm{FDB} \rightarrow \mathrm{FxB}+\mathrm{ATM}(+\mathrm{FRP})$ & & \\
\hline $\begin{array}{l}\text { Dissociation of ADP-Pi-actin from } \\
\text { pointed end } \\
\mathrm{FPP} \rightarrow \mathrm{FxP}+\mathrm{ADM}(+\mathrm{FRP})\end{array}$ & $k_{D I P P}$ & $0.8 \mathrm{~s}^{-1}[25,53]$ \\
\hline $\begin{array}{l}\text { Dissociation of ADP-actin from } \\
\text { pointed end }\end{array}$ & $k_{D I D P}$ & $0.27 \mathrm{~s}^{-1}[53]$ \\
\hline $\mathrm{FDB} \rightarrow \mathrm{FxB}+\mathrm{ADM}(+\mathrm{FRP})$ & & \\
\hline $\begin{array}{l}\text { Capping of the barbed end } \\
\mathrm{CBM}+\mathrm{FxB} \rightarrow \mathrm{CBF}\end{array}$ & $k_{A S C B}$ & $\begin{array}{l}3.0 \mu \mathrm{M}^{-1} \mathrm{~s}^{-1}[54] \\
8.0 \mu \mathrm{M}^{-1} \mathrm{~s}^{-1}[14]\end{array}$ \\
\hline $\begin{array}{l}\text { Capping of the pointed end } \\
\mathrm{CPM}+\mathrm{FxP} \rightarrow \mathrm{CPF}\end{array}$ & $k_{A S C P}$ & $\sim 0.25-1.0 \mu \mathrm{M}^{-1} \mathrm{~s}^{-1}[14]$ \\
\hline $\begin{array}{l}\text { Uncapping of the barbed end } \\
\mathrm{CBF} \rightarrow \mathrm{CBM}+\mathrm{FxB}\end{array}$ & $k_{D I C B}$ & $\begin{array}{l}4.0 \times 10^{-4} \mathrm{~s}^{-1}[54] \\
4.2 \mathrm{~s}^{-1}[14]\end{array}$ \\
\hline $\begin{array}{l}\text { Uncapping of the pointed end } \\
\mathrm{CPF} \rightarrow \mathrm{CPM}+\mathrm{FxP}\end{array}$ & $k_{D I C P}$ & No data \\
\hline $\begin{array}{l}\text { Association of formin to barbed end } \\
\text { FOM }+ \text { FxB } \rightarrow \text { FOF }\end{array}$ & $k_{A S F B}$ & No data \\
\hline $\begin{array}{l}\text { Detachment of formin from barbed } \\
\text { end } \\
\text { FOF } \rightarrow \text { FOM + FDB }\end{array}$ & $k_{D I F B}$ & $7.5 \times 10^{-4} \mathrm{~s}^{-1}[11]$ \\
\hline $\begin{array}{l}\text { Formin-initiated association at } \\
\text { barbed end }\end{array}$ & $k_{\text {FASB }}$ & $\begin{array}{l}\text { For mDia formin in the presence } \\
\text { of profilin } 45-110 \mu \mathrm{M}^{-1} \mathrm{~s}^{-1}[11,12]\end{array}$ \\
\hline $\mathrm{FOF}+\mathrm{ATM} \rightarrow \mathrm{FOF}+\mathrm{ADF}$ & & Without profilin $-9 \mu \mathrm{M}^{-1} \mathrm{~s}^{-1}[12]$ \\
\hline $\begin{array}{l}\text { Arp2/3 association to the } \mathrm{F} \text {-actin } \\
\mathrm{AxF}+\mathrm{ARM} \rightarrow \mathrm{AxF}+\mathrm{ARF}+\mathrm{FRB}\end{array}$ & $k_{\text {ASRT }}$ & $5.4 \times 10^{-4} \mu \mathrm{M}^{-3} \mathrm{~s}^{-1}$ \\
\hline $\begin{array}{l}\text { Dissociation of the Arp 2/3 from } \\
\text { the pointed end }\end{array}$ & $k_{\text {DIRP }}$ & $0.0018 \mathrm{~s}^{-1}[14]$ \\
\hline $\mathrm{FRP}[+\mathrm{ARF}] \rightarrow \mathrm{ARM}+\mathrm{FxP}$ & & \\
\hline Fragmentation: ${ }^{\wedge} \rightarrow \mathrm{FyB}+\mathrm{FxP}$ & $\mathrm{k}_{\mathrm{FRGM}}$ & No data \\
\hline Annealing: $\mathrm{FyB}+\mathrm{FxP} \rightarrow^{\wedge}$ & $\mathrm{k}_{\mathrm{ANNL}}$ & No data \\
\hline ATP-hydrolysis: ATF $\rightarrow$ APF & $k_{\text {TTOP }}$ & $0.3 \mathrm{~s}^{-1}[42,55]$ \\
\hline Phosphate release: $\mathrm{APF} \rightarrow \mathrm{ADF}$ & $k_{P T O D}$ & $0.0026 \mathrm{~s}^{-1}[43,55]$ \\
\hline $\begin{array}{l}\text { Recharge of monomeric actins in } \\
\text { the pool } \\
\text { ADM } \rightarrow \text { ATM }\end{array}$ & $k_{\text {Dтот }}$ & $\sim 20$ (pro) $\mathrm{s}^{-1}[56]$ \\
\hline
\end{tabular}

For abbreviations of reagents see Abbreviations.

*An experimental and theoretical study, reported in [52], indicates that the trimer is the critical nucleus for spontaneous nucleation of actin monomers. The spontaneous actin nucleation can be well approximated by a third order nucleation step for an actin monomer concentration higher or equal to $2 \mu \mathrm{M}$ [14]]. Other hypotheses on mechanism of spontaneous filament nucleation, including explicit, coupled nucleation steps may be implemented within the frame of our simulation scheme.

**FxB stands for the types of barbed ends: FTB, FPB, FDB and FRB; FyB for FTB, FPB, FDB; FxP for FTP, FPP and FDP and AxF for ATF, APF and ADF; ^ denotes the position between two actin protomers in filament. 
Eq. (1) is applied if only one molecule of each reacting reagent is simulated in the $i$-th reaction. If more than one molecule of at least one reagent participates in the reaction, as, for instance, in the formin-initiated nucleation, then Eq. (2) can be calculated based on an analogy with [37].

$a_{i}=k_{i}\left(10^{-6} N_{A} V\right)^{1-\eta_{i}} \prod_{j=1}^{n_{i}}\left(\prod_{l=1}^{m_{i}^{j}}\left(N_{j}-l+1\right)\right)$

where

$m_{i}^{j}$ is the number of molecules of $j$-th reagent, required for occurrence of $i$-th reaction.

$\eta_{i}=\sum_{j=1}^{n_{i}} m_{i}^{j}$ is the total number of molecules, participating in the $i$-th reaction. For instance for the formin-initiated nucleation $n_{F N U C}=2$ (ATM and FOM are the reagents for this reaction), $m_{F N U C}^{A T M}=3$, $m_{F N U C}^{F O M}=1$ and $\eta_{F N U C}=4$.

The selection of the type of the next reaction is performed in block 3 using a random number generator so that the probability to select $r$-th reaction is proportional to the value of $a_{r}$, where $r$ is the index running for 28 reactions $\{S N U C, C B N U, \ldots, D T O T\}$.

The time towards the reaction $\tau$ is calculated in block 4, using an assumption that the flow of reaction-events is a Poisson flow [34], and, therefore, the times between events are exponentially distributed. Using the method of inverse functions, the time $\tau$ can be calculated as

$\tau=-\left(\sum_{i} a_{i}\right)^{-1} \ln (\xi)$

where $\xi$ is the uniformly distributed random number from the interval $(0,1)$.

The $f$-th filament, to which the reaction will be applied, is randomly selected among the total number $n_{F}$ of filaments in block 5 using a new realization of a random number generator. The filament selection procedure is a numeric routine governed by a complex density probability function of the numbers of branches, pointed and barbed ends for the current state of filaments distribution. If the annealing reaction is gambled then two new random numbers are generated. This step is not needed in case of the nucleation reactions.

In block 6 the $p$-th filamentous unit in the $f$-th filament is generated, using a new realization of a random number generator, on which a reaction either of hydrolysis, branching, or fragmentation occurs. This step is ignored in case of nucleation, association, dissociation, capping, uncapping, annealing reactions.

The reaction is performed in block $\mathbf{7}$ in accordance with a formal scheme listed in Table 1. Each reaction results in a modification of the number of molecules and structure of one of the filaments. Exceptions to this are the hydrolysis and recharging reactions, which affect only structure of proteins. Nucleation and fragmentation reactions result in appearance of new filaments. Annealing reaction leads to the disappearance of one of the filaments. Branching reaction yields a new filament branch. When one type of the actin-monomer dissociation reactions occurs, the length of the filament is checked. If it contains less then 3 actin protomers - the filament dissociates, releasing all attached proteins (i.e. formin, capping proteins). The ATPrelated type of F-actin ends (FTB, FDB, FTP, FDP) can be changed after association and dissociation reactions.

Finally, the systems time $t$ is increased by $\tau$ (block 8), and if the simulation time is less than the predefined maximal simulation time $t_{\max }$ (block 9), the algorithm returns to block 2. Otherwise simulation stops in block 10, providing a list of output characteristics, i.e. evolution of concentrations in time, the distribution of filament lengths, and actin nucleotide states in filaments.

\subsection{Non-structurally-resolved filament (nSRF) and structurally-resolved} filament (SRF) models

We developed two variants of structural representations of actin filaments: the non-structurally-resolved filament (nSRF) model and the structurally-resolved filament (SRF) model.

In the nSRF model a filament is represented by the number of ATP-containing actins (ATF), the number of ADP-Pi-containing actins (APF), the number of ADP-containing actins (ADF) and by type and state of the barbed and pointed ends. Selection of the state of actins (ATF/APF/ADF) bound to filament ends is performed as follows. The state of a last attached protein is stored in a model parameter called as "state of barbed/pointed end". It thus defines the states of the corresponding ends of the filament, i.e. FTB/FTP, FPB/FPP, FDB/FDP, FRB/FRP, CBF/CPF, or FOF. After dissociation of a monomer from the filament, the state of the next filament boundactin monomer, that will define a new type of end, is selected preferably from ATF actins. If no ATF actins are left, the filament end switches into ADP-Pi- (preferably) or ADP-related form. The SRF model is similar to the nSRF model but is additionally represented by the bidirectional list of actin types (ATF/APF/ADF) and actinaccessory proteins ( $\mathrm{CBF} / \mathrm{CPF} / \mathrm{FOF} / \mathrm{ARF})$, which defines mutual positions of protomers in the filament. During filament growth, a marker is stored in the bidirectional list to define ATP/ADP-Pi/ADP-state of each actin or subunit and/or actin-associated protein in filament. The list has two access points - pointers to the barbed and pointed ends. This organization permits swift addition/removal of monomers at the ends and splitting/merging of lists for fragmentation/ annealing reactions. When Arp2/3 induced-branching is included, the pointers to 'mother' and 'daughter' filament branches are stored in the SRF and nSRF models. The SRF model also stores positions of attachment points on the 'mother' filament or branch regarding the pointed end.

Using the SRF model is more time-consuming due to processing the bidirectional list. The nSRF model is aimed to speed up the simulation by simplifying the representation of filaments. The SRF model is more accurate than the nSRF model and, by accounting for mutual positions of ATP- and ADP-actins in filaments as described above, it gives a powerful opportunity for the evaluation of nucleotide compositions without any restrictions and conditions imposed on the distribution of filament lengths. By using the SRF model one is additionally able to check: (i) depolymerization of preformed actin filaments networks, (ii) filament severing, for example, by cofilins, which have a higher affinity for ADP-actin [38], (iii) other polymerization processes taking in account the nature of the nucleotide such as the Arp2/3 association and side branch initiation which is preferable occurring at an ATP-charged protomer.

\subsection{Error analysis}

Stochastic deviations in the simulated results, yielding asymptotic solutions of the investigated processes, are inherent to the Monte Carlo simulation technique. In addition, powerful computational facilities are required. Increasing a volume $V$ and averaging the results of $n_{A}$ independent simulations help to reduce the stochastic deviation. Therefore, we developed a verification rule for an optimal selection of the simulation volume $V$ and the number of averaging $n_{A}$ (see Appendix A). An increase of $n_{A}$ is preferable over an increase of $V$. The latter has a bottom cut-off to avoid any discontinuity at low concentrations and rare reactions. We estimated a possibly minimal volume needed for analysis of actin polymerization processes. Assume that the minimal number of reacting molecules is equal 20. If the minimal initial concentration of a reagent is $1 \mathrm{nM}$ then the minimal volume becomes $V_{\min } \approx 33 \mu \mathrm{m}^{3}$. If the polymerization starts from a low-rate nucleation with the rate constant of $\sim 10^{-9}-10^{-8}$, 
the selection of a larger volume of $V>125 \mu \mathrm{m}^{3}$ may, however, be required.

\section{Additional experimental procedures}

The developed models were evaluated for analysis of experimental data from fluorescence pyrene-actin polymerization assays. Below we used data reported in literature [14] or generated as described in 3.1.

3.1. Actin polymerization assays using proteins purified from tissues or produced in E. coli

Actin was prepared from rabbit skeletal muscle [39] and further purified using Sephacryl S-300 (Pharmacia) in G-buffer [5 mM Tris- $\mathrm{HCl}, \mathrm{pH}$ 7.7, $0.1 \mathrm{mM} \mathrm{CaCl}_{2}, 0.2 \mathrm{mM}$ ATP, $0.2 \mathrm{mM}$ dithiothreitol (DTT), $0.01 \% \mathrm{NaN}_{3}$ ]. Actin was labelled with Pyrene on Cys374 following Kouyama and Michashi [40]. To produce recombinant formin FH1-FH2 domain, the corresponding mouse formin cDNA (mDia-1) was cloned into the pQE30 vector (Qiagen). The production of the His tagged-FH1-FH2 domains was carried overnight at $16{ }^{\circ} \mathrm{C}$, after induction with $1 \mathrm{mM}$ IPTG. Proteins were purified from bacterial lysates using Talon ${ }^{\circledR}$ Metal Affinity Resin (Clontech) and eluted by $500 \mathrm{mM}$ Imidazole. The His taggedFH1-FH2 protein was dialysed against $100 \mathrm{mM}$ Tris pH 7.5; $100 \mathrm{mM}$ $\mathrm{KCl} ; 1 \mathrm{mM}$ DTT.

Polymerization of pure actin ( 3 or $2 \mu \mathrm{M}, 20 \%$ pyrene-labeled, in $\mathrm{G}$-buffer) or actin in the presence of formin, was induced by addition of $\mathrm{KCl}$ and $\mathrm{MgCl}_{2}$ to final concentrations of 100 and $1 \mathrm{mM}$, respectively. The fluorescence increase, proportional to filament formation, was followed as function of time using a F-4500 fluorimeter (Hitachi) (excitation at $365 \mathrm{~nm}$ and emission at $388 \mathrm{~nm}$ ). Experimentally obtained fluorescence data were corrected for background fluorescence by subtraction of the constant, equal to fluorescence at $t=0 \mathrm{~s}$. Subsequently, the fluorescence units were rescaled, to fit simulated concentrations. Several studies have shown that the pyrene fluorescence intensity of ADP F-actin is substantially stronger than that of ATP F-actin $[23,41]$. Consequently the relative amounts of ATP F-actin and ADP F-actin has been included in correlating pyrene intensity and F-actin concentration. In this work, the best quality of fit to experiments, giving the minimum error (Appendix A, Eq. B1), was obtained for a ratio of 0.5 of F-ATP to FADP-actin.

\subsection{Model validation}

First, the source code was verified (see Appendix A). Second, we tested and validated the Monte Carlo model by comparison with synthetic theoretical data. A system of corresponding differential equations was used for a number of pre-selected sets of biochemical parameters published elsewhere. Third, we compared the computational efficiency and agreement of various actin filament models: nonstructurally-resolved (nSRF) and structurally-resolved filament (SRF) models. Finally, we applied our model for the analysis of pyrene-actin based fluorescence actin-polymerization experiments: i) actin polymerization, ii) actin polymerization in the presence of actin-capping protein [14], or iii) formin.

\section{Results and discussion}

\subsection{Strategy for model evaluation and analysis}

We evaluated the performance of the developed models on wellcharacterized theoretical and published data. We considered fundamental reactions including monomer assembly into filaments, filament end capping and assembly of actin oligomer nuclei by capping protein or formin. The complete list of the reactions events simulated is shown in Fig. 1. Our simulations are performed for $V=125 \mu \mathrm{m}^{3}$ and $n_{A}=50$. For computational reasons and taking into account its minor effect on the systems considered in our tests we do not include in the simulations the reactions associated with the ADP-Pi "intermediate" nucleotide state. Instead, we approximated the filament aging reaction (hydrolysis of ATP in filaments) with the overall rate constant $0.0007 \mathrm{~s}^{-1}$, which is assumed to be a lowest limit for an approximative two-step hydrolysis rate constant (including phosphate release), and is a product of rate constants for hydrolysis $0.3 \mathrm{~s}^{-1}$ [42] and phosphate release 0.0026-0.004 $\mathrm{s}^{-1}$ $[43,44]$.

We compared the nSRF model with a deterministic kinetic model for the simplest situation, in which the behaviour of the actin system can be described by a system of differential equations. Such deterministic models have been used to describe actin polymerization kinetics (see e.g. $[13,14,45,46])$. Subsequently, we compared and evaluated simulations obtained with the SRF and nSRF models, a key step towards the model validation. First, agreement between two independently implemented models gives a good confidence that no technical or logical errors were made during programming of the modelling algorithms. Second, this comparison helps to investigate
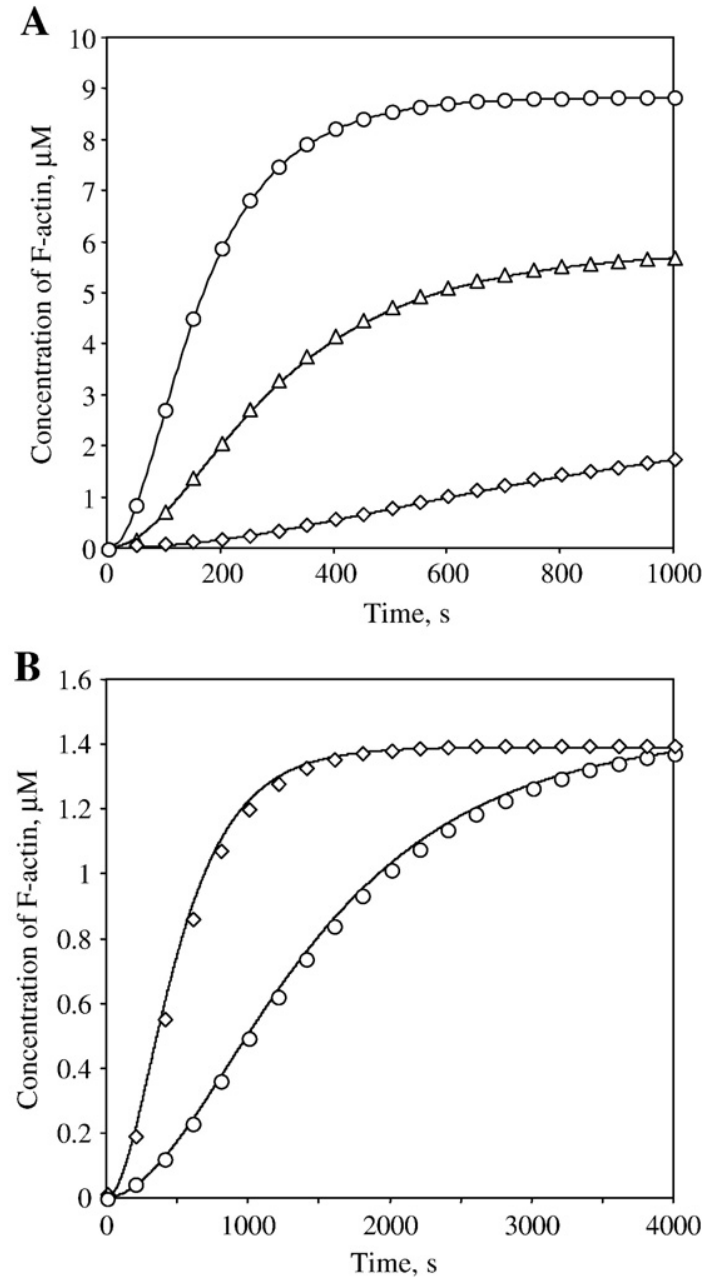

Fig. 3. Predicted concentrations of F-actin by the nSRF (symbols) and analytical models (lines) for (A) actin polymerisation and (B) actin polymerisation in presence of a filament barbed end capping protein, $(\mathrm{A})[\mathrm{ATM}]_{\mathrm{t}=0}=3,6$ and $12 \mu \mathrm{M}$ (bottom, middle and top curves), $k_{S N U C}=10^{-8} \mu \mathrm{M}^{-2} \mathrm{~s}^{-1}$, (B) $[\mathrm{ATM}]_{\mathrm{t}=0}=2 \mu \mathrm{M},[\mathrm{CBM}]_{\mathrm{t}=0}=0.1 \mu \mathrm{M}$ (top curve) and $0.01 \mu \mathrm{M}$ (bottom curve), $k_{C B N U}=10^{-5} \mu \mathrm{M}^{-3} \mathrm{~s}^{-1}$. If a parameter is not mentioned specifically, the value from Table 1 is used. 
the application range of the nSRF model, i.e. to determine when the simplifications of the nSRF model are acceptable and do not lead to significant changes in simulation results. Finally, to further evaluate the robustness of the developed models, we applied the simulation models to some selected experimental data and evaluated how reagents affect the reactions.

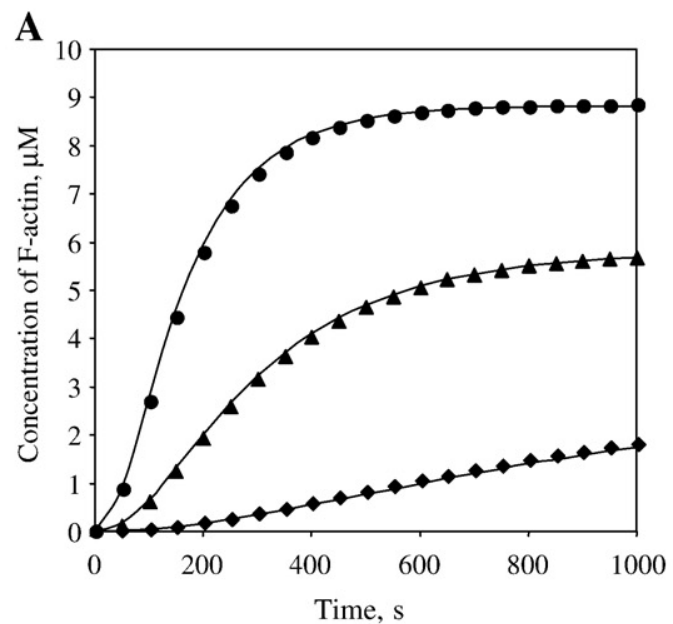

B

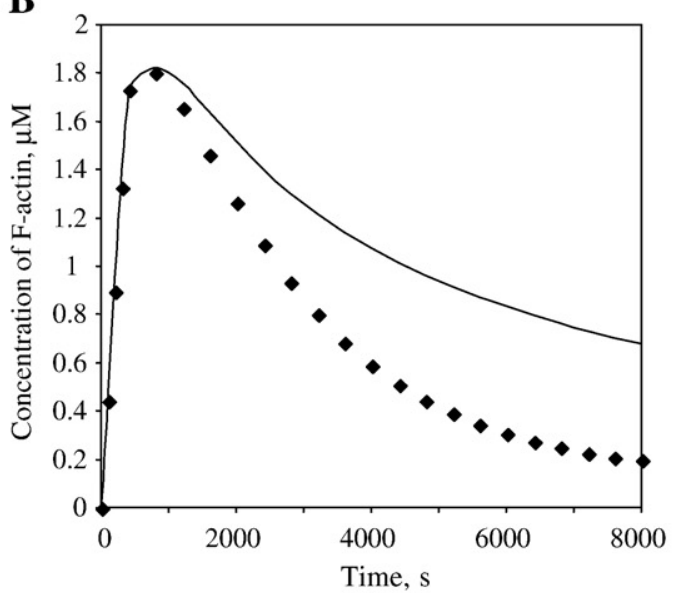

C

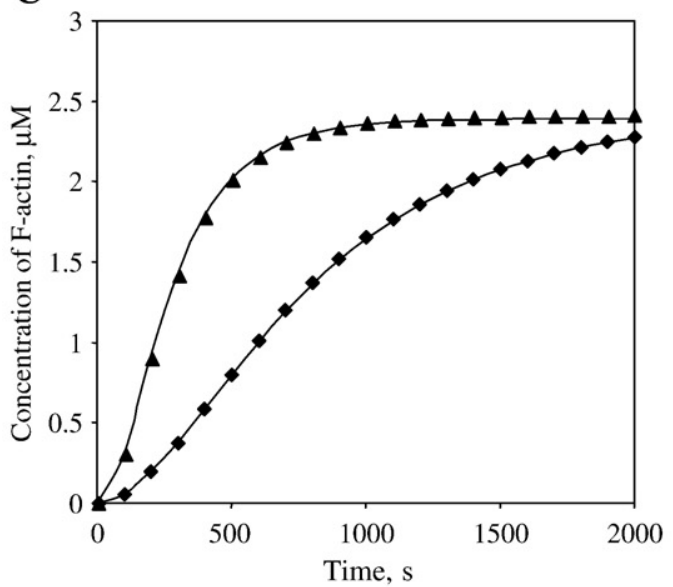

D
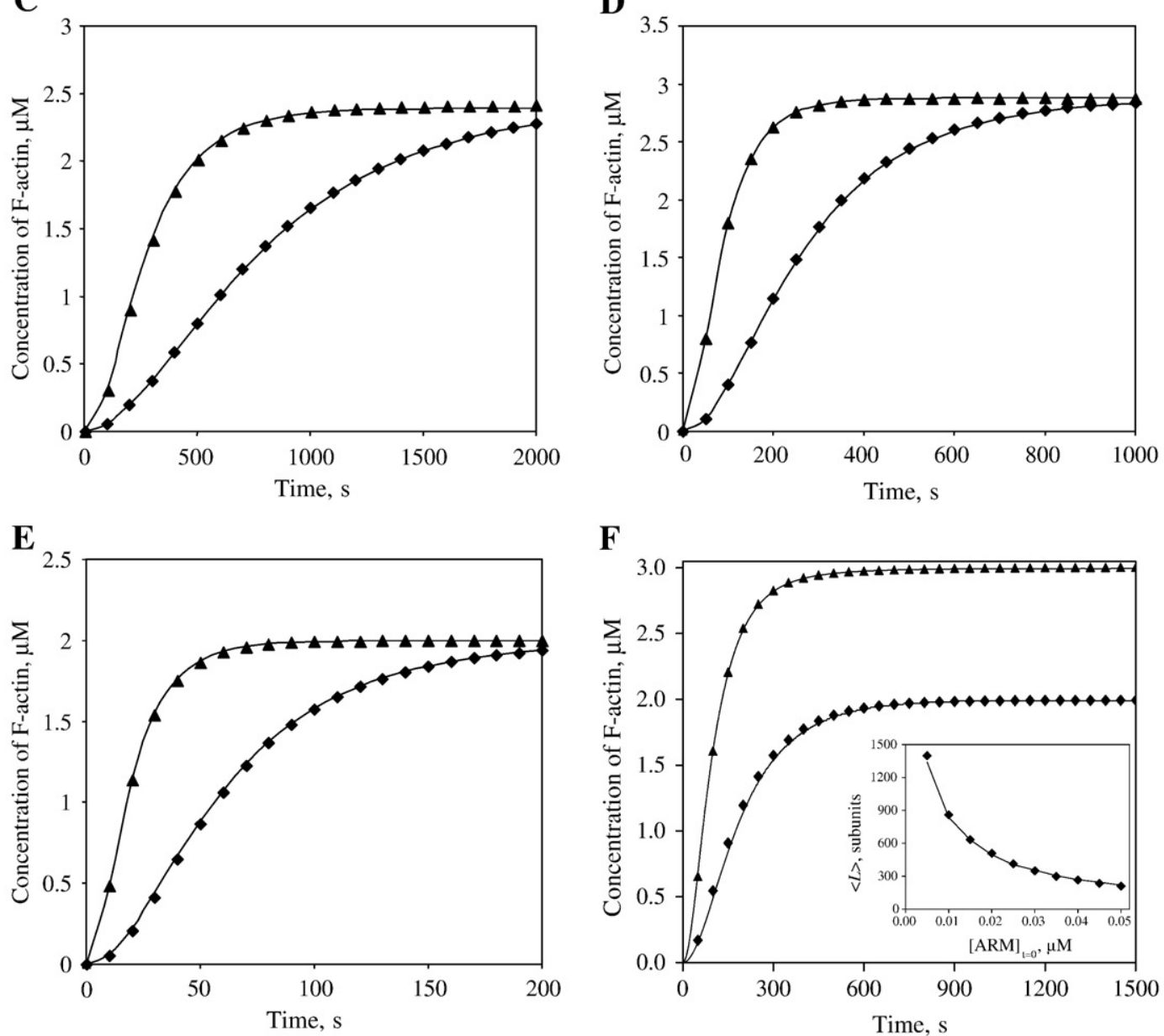

F

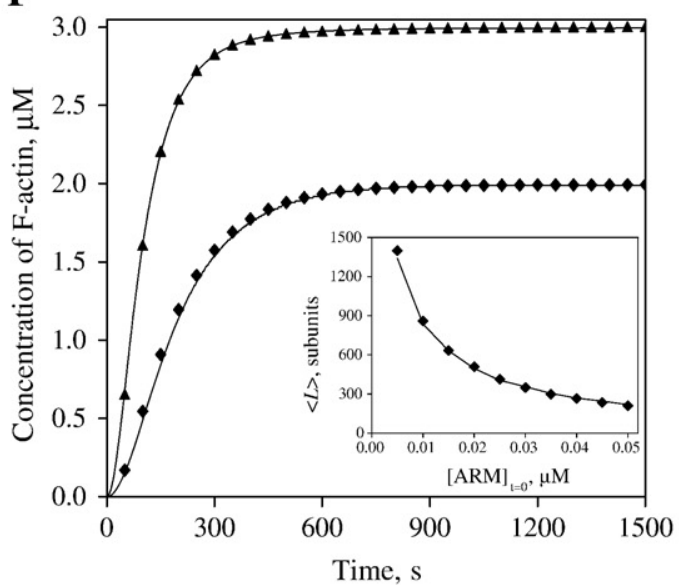

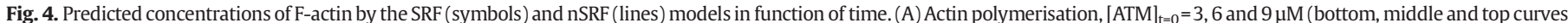

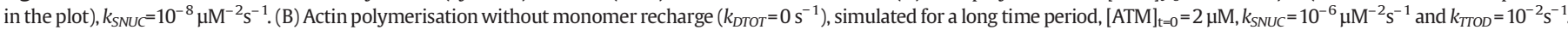

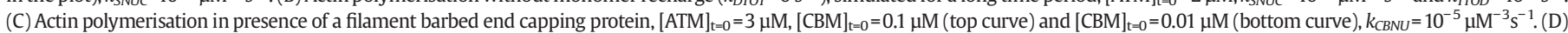

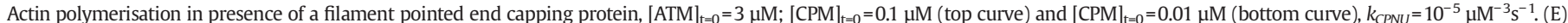

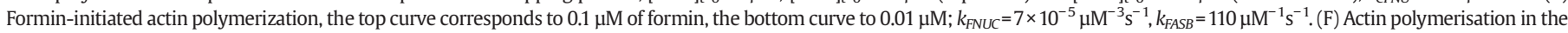

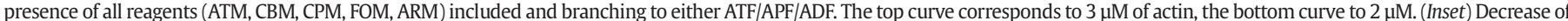

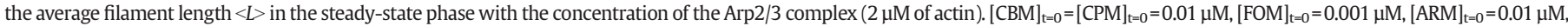

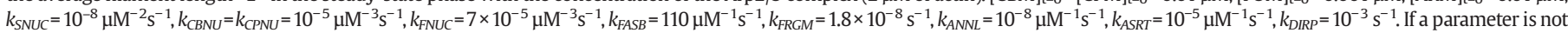
mentioned specifically, the value from Table 1 is used. 


\subsection{Evaluation of the developed models by analytical solutions}

\subsubsection{Polymerization reaction of actin}

Actin assembly is a simple model system. The concentration of F-actin only slowly increases in the early phase (the so-called lag phase) due to slow spontaneous nucleation. Then a period of fast elongation follows, during which the concentration of F-actin increases more rapidly. Consequently globular actin decreases and asymptotically approaches to the so-called critical concentration $C_{c}$. The critical concentration, which is independent from the initial actin concentration, can be calculated via association/dissociation rates, Eq. (4)

$\mathrm{C}_{\mathrm{c}}=\left(\mathrm{k}_{\mathrm{DITB}}+\mathrm{k}_{\mathrm{DITP}}\right) /\left(\mathrm{k}_{\mathrm{ASTB}}+\mathrm{k}_{\mathrm{ASTP}}\right)$.

We initially assume that no ATP-hydrolysis occurs in a system, i.e. no aging of F-actins, and, thus, only ATM and ATF actins exist in this system. This assumption is needed to build the system of the analytical equations (see Appendix A) and not a limitation linked to the presented simulation (see Section 4.3.1). The rate constants (for references see Table 1) used in modelling are: $k_{S N U C}=10^{-8} \mu \mathrm{M}^{-2} \mathrm{~s}^{-1}$, $k_{\text {ASTB }}=11.5 \mu \mathrm{M}^{-1} \mathrm{~s}^{-1}, k_{\text {ASTP }}=1.3 \mu \mathrm{M}^{-1} \mathrm{~s}^{-1}, k_{\text {DITB }}=1.4 \mathrm{~s}^{-1}, k_{\text {DITP }}=0.8 \mathrm{~s}^{-1}$. The nSRF model was launched and tested at three initial actin concentrations $[\mathrm{ATM}]_{\mathrm{t}=0}=3,6$ and $12 \mu \mathrm{M}$. Predicted F-actin concentrations obtained either by the nSRF model or by the analytical model (Eq. (C1), Appendix A) for the actin systems with different actin concentrations are plotted in Fig. 3A and indicate a good agreement between simulated and analytical data.

4.2.2. Actin polymerization reaction in the presence of capping protein

Addition of barbed-end capping protein in the system above introduced two effects: i) increase of the nucleation rate and ii) inhibition of further elongation from the barbed end by capping. The analytical model for actin polymerization in the presence of a barbed-end capping protein was reported in [14] and briefly given in Appendix A (Eq. (C2)). Again, no aging of F-actins is assumed (this is necessary to avoid tracking the ATP/ADP state of the ends for each separate filament). The numerical experiments were launched with the rate constants: $k_{S N U C}=10^{-8} \mu \mathrm{M}^{-2} \mathrm{~s}^{-1}$,

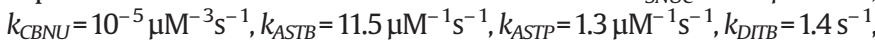
$k_{D I T P}=0.8 \mathrm{~s}^{-1}, k_{A S C B}=3 \mu \mathrm{M}^{-1} \mathrm{~s}^{-1}, k_{D I C B}=4 \times 10^{-4} \mathrm{~s}^{-1}$ (cf. Table 1). Predicted concentrations of F-actin by the nSRF (circles, diamonds) and analytical model (lines) for the initial protein concentrations $[A T M]_{t=0}=2 \mu \mathrm{M}$, and $[\mathrm{CBM}]_{\mathrm{t}=0}=0.1,0.01 \mu \mathrm{M}$ are plotted in Fig. 3B. The fact that the results of simulated and analytical models of actin polymerization, with or without capping protein, are in good agreement validates the developed simulation model.

A broader range of examples and tests of the models is shown in Appendix A (Fig. S1-S2). Fig. S1 demonstrates that simulations that predict the average filament length and the filament length distribution at steady-state changed drastically in presence of fragmentation and annealing, in line with [25]. Fig. S2 shows a simulation of overshoot occurrence under various conditions of polymerization kinetics.

\subsection{Comparison of the nSRF and SRF models}

\subsubsection{Polymerisation of actin in the absence of other proteins}

A major difference between SRF and the nSRF model (and, $a$ fortiori, previous analytical models) is, that the former inherently incorporates the possibility of aging of F-actin. This is now taken into account and simulated as a stochastic reaction with the rate constant $0.0007 \mathrm{~s}^{-1}$. The SRF model combines information about type and position of each monomer in the filament, whereas in the nSRF model it is assumed that ATP-containing F-actins (ATF) are allocated near the filament ends, and ADP-actins (ADF) in the middle. The latter, rather rough approximation of the F-actin aging mechanism may result in biased models solutions, in particular for a time period longer than
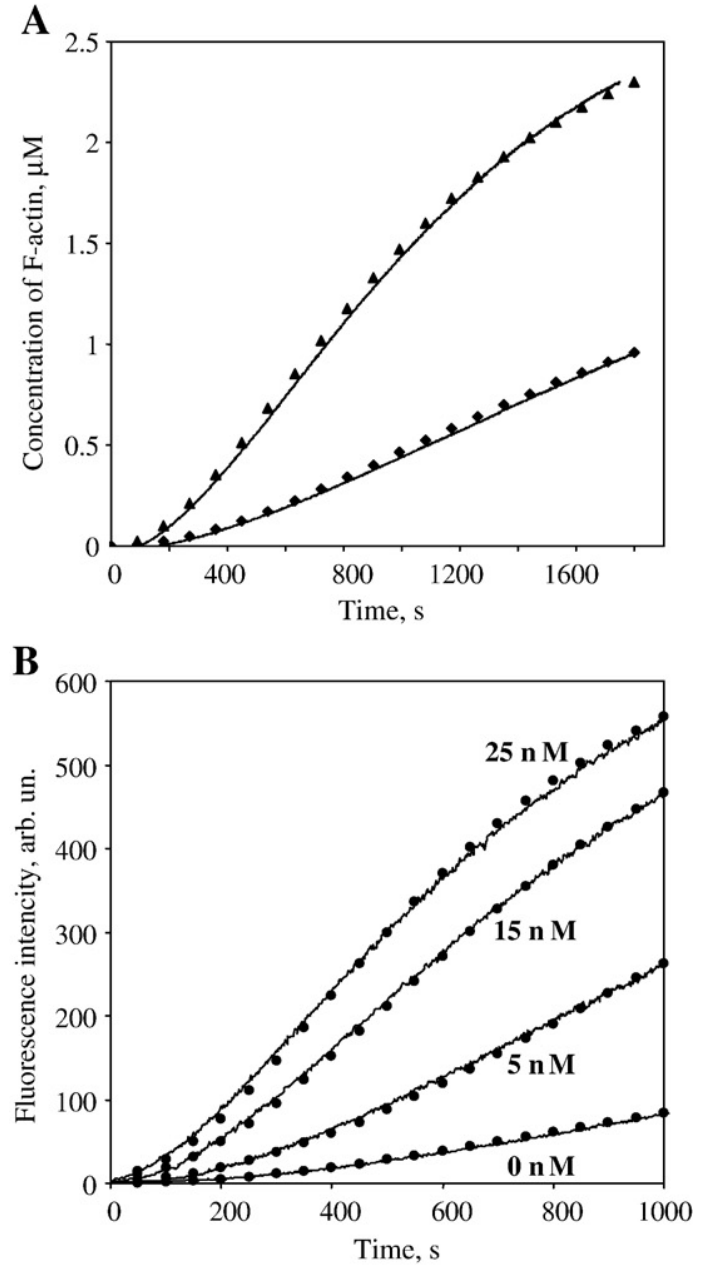

C

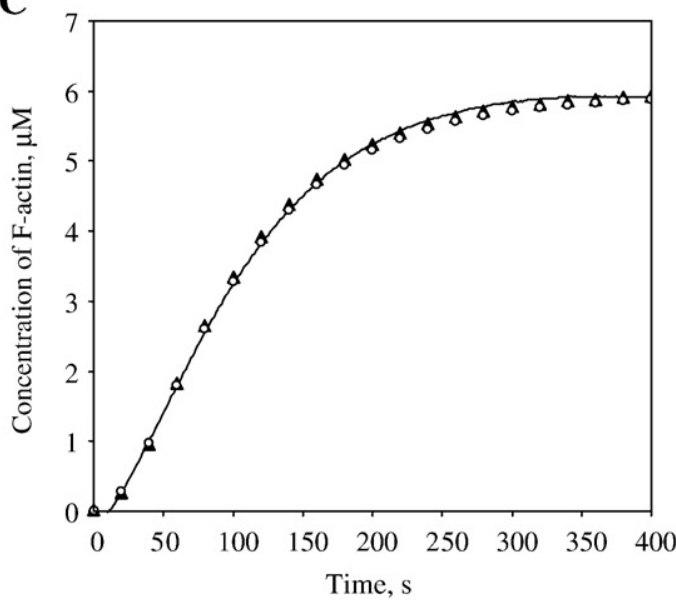

Fig. 5. Comparison of the simulation results (symbols) with experimentally obtained data (solid lines). (A) Actin assay with $[\mathrm{ATM}]_{\mathrm{t}=0}=2 \mu \mathrm{M}$ (bottom curve) and $3 \mu \mathrm{M}$ (top curve). Experimental fluorescence data were corrected for background and normalized by amplitude to fit simulated concentrations. During simulation $k_{S N U C}=4.1 \times 10^{-9} \mu^{-2} \mathrm{~s}^{-1}$, the other rates are the same as listed in Table 1. (B) Experimental data showing the effect of capping-protein nucleation from [14] (solid lines). Experimental assays contain $2 \mu \mathrm{M}$ of actin and various concentrations of barbed-end capping protein: $[\mathrm{CBM}]_{\mathrm{t}=0}=0,5,15$ and $25 \mathrm{nM}$. (C) Formin-initiated actin polymerization. Here the comparison of the experimental fluorescence data (solid line), normalized by amplitude, and simulation results for two models of formin-actin polymerization (symbols) are given. The experimental sample contains $[\mathrm{ATM}]_{\mathrm{t}=0}=6 \mu \mathrm{M}$ and $[\mathrm{FOM}]_{\mathrm{t}=0}=0.2 \mu \mathrm{M}$. Black triangles corresponds to the model with $k_{F A S B}=9 \mu \mathrm{M}^{-1} \mathrm{~s}^{-1}$ and $k_{F N U C}=4.7 \times 10^{-7} \mu \mathrm{M}^{-3} \mathrm{~s}^{-1}$. White circles corresponds to the model with $k_{F A S B}=90 \mu \mathrm{M}^{-1} \mathrm{~s}^{-1}$ and $k_{F N U C}=4.7 \times 10^{-8} \mu \mathrm{M}^{-3} \mathrm{~s}^{-1}$. 
$1000 \mathrm{~s}$, assuming a slow rate constant $\sim 10^{-3} \mathrm{~s}^{-1}$ for the hydrolysis reaction.

Fig. 4A plots the predicted concentrations of F-actin resulting from the SRF (symbols) and nSRF (lines) models for three initial concentrations of actin $[\mathrm{ATM}]_{\mathrm{t}=0}=3,6$ and $9 \mu \mathrm{M}$. The concentration of F-actin slowly increases in early phase, consistent with the experimentally observed lag phase (see e.g. Fig. 5B), due to slow spontaneous nucleation $\left(k_{S N U C}=10^{-8} \mu \mathrm{M}^{-2} \mathrm{~s}^{-1}\right)$. During fast elongation the concentration of F-actin increases, consequently globular actin decreases and asymptotically approaches the critical concentration $C_{c}$ which here is $0.17 \mu \mathrm{M}$. Therefore, the concentration of F-actin asymptotically approaches values of $2.83,5.83$ and $8.83 \mu \mathrm{M}$ (Fig. 4A).

During nucleation and elongation the rates of ADP-actin association and dissociation do not play an important role, because of fast ATP-actin recharge in the monomer pool and significant prevailing of the ATP-actin association reactions. At this stage no deviation can be detected when comparing both models, therefore the nSRF model can be used instead of SRF to predict systems behaviour in the nonequilibrium elongation phase of the filament. The simulation time required by the nSRF model for an actin system is about 30 times lower than that of the SRF model.

To evaluate the effect of ATP hydrolysis and filament aging, we varied the model parameters. We assumed that no actin recharge occurred and, to make the effect of aging more pronounced, we increased the aging rate up to one hundred times, and increased the rate of spontaneous nucleation up to $k_{S N U C}=10^{-6} \mu \mathrm{M}^{-2} \mathrm{~s}^{-1}$. Since the effect may appear only when association no longer prevails over dissociation, a long time period of $t_{\max }=8000 \mathrm{~s}$ was considered. The simulation results of the system for $[\mathrm{ATM}]_{\mathrm{t}=0}=2 \mu \mathrm{M}$ are shown in Fig. 4B. No deviation was observed for the period of filament elongation, however, the subsequent late dissociation phase significantly differed for the two models. This deviation occurred even if actin ATP-recharge was switched on, however to a much lesser degree (data not shown).

This result indicates that when ATP-actin recharge exists in the system, the simplified nSRF model can be used during the periods of lag-phase and fast filament elongation. To further confirm this result, we considered more complex systems including actin regulatory proteins that cap or nucleate actin polymerization.

\subsubsection{Actin polymerization reactions in the presence of end cappers or} nucleating proteins

A barbed-end capping protein initially promotes assembly of oligomeric actin nuclei and subsequently inhibits elongation of the filament barbed end by remaining associated with it $[14,17]$ thereby it can affect the critical concentration. Previous reports on modelling of actin polymerization suggested that the nucleation reaction involves 6 actin monomers, without however excluding that nucleation with 3 monomers might be also possible [14]. We used 3 actin molecules and an attached capping protein as a filament nucleus for simplification (see reaction with the rate constant $k_{C B N U}$ in Table 1 ).

Fig. $4 \mathrm{C}$ shows the results of the simulation for $3 \mu \mathrm{M}$ actin in the presence of 0.01 or $0.1 \mu \mathrm{M}$ barbed-end capping protein, respectively. The nucleation rate of the capping-protein was set to $10^{-5} \mu \mathrm{M}^{-3} \mathrm{~s}^{-1}$. Nucleation activity of the capping protein considerably increased the rate at which actin filaments formed, as a function of capping protein concentration. The steady-state F-actin concentration was, however, higher for lower capping protein concentration: $2.51 \mu \mathrm{M}$ for $[\mathrm{CBM}]_{\mathrm{t}=0}=0.01 \mu \mathrm{M}$ vs. $2.41 \mu \mathrm{M}$ for $[\mathrm{CBM}]_{\mathrm{t}=0}=0.1 \mu \mathrm{M}$. No deviation could be observed between the results of SRF (symbols) and nSRF (lines) model for the time between 0 and $2000 \mathrm{~s}$ (Fig. 4C). For longer times slight deviations appeared (not shown), as observed for the system with actin alone.

Next, we analyzed the effect of pointed-end cappers at similar concentrations. We assumed that this protein had similar properties (including rate constants) as the barbed-end capping protein, with the difference that it attached to the pointed end of the filament. Fig. 4D revealed a good agreement between models. The time scale of the plot was between 0 and $1000 \mathrm{~s}$, since, as was expected, the F-actin elongation rate was approximately 3 times faster than that in the presence of the barbed-end capping protein. Moreover, the steadystate concentrations of F-actin were around $2.9 \mu \mathrm{M}$ (for both CPM concentrations) due to the difference in critical concentrations of monomeric actin for free barbed $(\sim 0.12 \mu \mathrm{M})$ and pointed $(\sim 0.61 \mu \mathrm{M})$ ends.

\subsubsection{Formin-mediated effects on actin polymerization system}

There are two main functions of formin in actin polymerization: it assembles actin and acts as a processive motor of filament elongation. In the presence of profilin, formin effectively generates long ADP-rich filaments, as was reported in [11]. The elongation rate in this situation may reach values of $110 \mu \mathrm{M}^{-1} \mathrm{~s}^{-1}$. In the absence of profilin, the elongation is less efficient, with a rate constant of $9 \mu \mathrm{M}^{-1} \mathrm{~s}^{-1}$ [12].

In our simulations we used the information from [11] and [13] to estimate formin-associated rate constants (see Table 1, reaction with rate constants $\left.k_{F N U C}, k_{F A S B}, k_{D I F B}\right)$. The rate of spontaneous association of formin to a free barbed-end was put arbitrary, $k_{A S F B}=3 \mu \mathrm{M}^{-1} \mathrm{~s}^{-1}$, however, this reaction does not demonstrate a high influence on the polymerization, because usually all free formins tend to nucleate new filaments. The result of the comparison of SRF and nSRF models for a $2 \mu \mathrm{M}$ actin assay is given in Fig. 4E. Note, that the time window of the simulation $[0 ; 200 \mathrm{~s}]$ is very different from the previous situations and that the speed of filament growth has increased significantly. For both formin concentrations tested, the final F-actin concentration is equal to $1.997 \mu \mathrm{M}$, calculated as extrapolation to longer times, with almost all actin molecules in the F-form.

\subsubsection{Effect of the full reaction set on actin polymerization system}

We theoretically compared the simulation results for the condition in which all reagents and reactions are present (Table 1, Fig. 1). Selected initial concentrations are: $[\mathrm{ATM}]=2$ and $3 \mu \mathrm{M}$; $[\mathrm{CBM}]_{\mathrm{t}=0}=0.01 \mu \mathrm{M}$, $[\mathrm{CPM}]_{\mathrm{t}=0}=0.01 \mu \mathrm{M},[\mathrm{FOM}]_{\mathrm{t}=0}=0.001 \mu \mathrm{M},[\mathrm{ARM}]_{\mathrm{t}=0}=0.01 \mu \mathrm{M}$. This is a very complex situation since barbed end capping protein interferes with formin and formin lowers the binding of capping protein to barbed ends by 100 fold without, however, competing for barbed end binding ([11], supplement data on gelsolin). In addition, several reactions including the Arp2/3 dependent branching reaction, change the number of (barbed) filament ends. Despite this complexity, the comparison of F-actin behaviour for two tested actin concentrations shown in Fig. 4F, demonstrates that the results for SRF and nSRF models were in a good agreement. Using our model, we can in addition investigate an effect of the branching reaction, in terms of the relevant rate constant and the concentration of the Arp2/3 complex, on the average filament length at steady state. We developed two mechanisms of the side branching: the Arp2/3 complex random nucleation to only ATF or to either ATF/APF/ADF. The latter case, despite not yielding a difference between nSRF and SRF models even at steady state, is needed to validate the simulation algorithms (Fig. 4F, inset). The average filament length in the steady-state phase decreases with the concentration of the Arp2/3 complex by both nSRF and SRF models - as is indeed expected from previous analytical calculations [47]. In Appendix A we show an additional simulation example demonstrating that the outcome of Arp2/3 - complex association and side branch initiation, which preferably occurs at an ATP-charged protomer, may differ for SRF and nSRF models (see Fig. S3 of Appendix A).

Based on the synthetic data we obtained for systems with increasing complexity and from comparing the two models where one model ignores the positional information of actin monomers in the filament, one is lead to conclude that unless the system is deprived of ATP-actin, both models yield the same result. This surprisingly suggests that the relative positions of ATP-actin and ADP-actin protomers within a filament are not important. One possible explanation is that the ATP 
hydrolysis rate does not influence the early time kinetics because it is very slow $\left(0.0007 \mathrm{~s}^{-1}\right)$. This interpretation explains why kinetic models without ATP hydrolysis are also successful. Once the system reaches the steady state phase, however, the nucleotide status may play a significant role for actin polymerisation (as demonstrated in Fig. 4B) for severing of filaments by cofilin (at ADP-actin protomers) or branching by Arp2/3 complex (at ATP-actin protomers as shown in Fig. S3, Appendix A). In this case the developed SRF will yield more accurate results.

Additional numerical tests on validation of the developed models, in particular, effects of capping protein concentration and of various rate constants on actin polymerization, are reported in Appendix A. Collectively, this leads to the following conclusions. Increasing the concentration of capping proteins leads to increase of the overall polymerization rate due to faster nucleation. Interestingly, if we exclude the nucleation activity of capping proteins, pointed-end capping proteins had no significant effect on actin polymerization kinetics. Second, we detected that already a moderate, twofold increase/decrease in monomer association/dissociation rates at the barbed ends strongly affects actin polymerization kinetics and almost no effect can be seen for the pointed ends. The simulation shows that increasing the nucleation rate results in a considerable rise in polymerized actin. Finally, variation in the aging rate does not modify the actin polymerization kinetics until steady-state is reached.

\subsection{Evaluation on experimental data}

To further evaluate the robustness of the developed models, we applied them to fitting of experimental data.

\subsubsection{Actin polymerization}

The simulation for 2 and $3 \mu \mathrm{M}$ of actin concentrations has been performed with the same nucleation rate $k_{S N U C}=4.1 \times 10^{-9} \mu \mathrm{M}^{-2} \mathrm{~s}^{-1}$. The results of the comparison are given in Fig. 5A. Possible inconsistency between simulated and experimental data would result in curvature difference. Simulated and experimental data were however in good agreement, although small non-significant differences could be observed ( $3 \mu \mathrm{M}$ of actin, top curve).

\subsubsection{Actin polymerization in the presence of capping proteins}

We next evaluated the effect of barbed end capping protein. We tested our models on data published previously in [14]. Keeping the actin concentration constant $(2 \mu \mathrm{M})$, the concentration of CBM was increased between 0 and $25 \mathrm{nM}$. The resulting change in pyrene fluorescence in time is given by solid lines in Fig. 5B. These experimental data and the simulation results, presented by symbols, highly correlated. The best fitting was obtained with the nucleation rate $k_{C B N U}=1.2 \times 10^{-5} \mu^{-3} \mathrm{M}^{-1}$

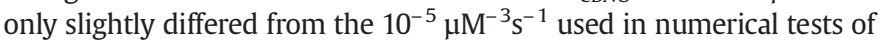
Sections 4.2.2-4.3.2.

\subsubsection{Formin-actin polymerization}

The experimental assay included $6 \mu \mathrm{M}$ actin and $0.2 \mu \mathrm{M}$ formin (in the absence of profilin). First, we fixed the formin-enhanced elongation rate of actin filament, taking the value of $k_{F A S B}=9 \mu \mathrm{M}^{-1} \mathrm{~s}^{-1}$ [12]. Then the nucleation rate was fitted. The best fit (Fig. 5C) was obtained for a nucleation rate constant of $k_{F N U C}=4.7 \times 10^{-7} \mu \mathrm{M}^{-3} \mathrm{~s}^{-1}$, which is $~ 10$ times higher than the value for spontaneous actin nucleation. We could get acceptable fittings with other combinations of values for $k_{F A S B}$ and $k_{F N U C}$, for example, with $k_{F A S B}=90 \mu \mathrm{M}^{-1} \mathrm{~s}^{-1}$ and $k_{F N U C}=4.7 \times 10^{-8} \mu \mathrm{M}^{-3} \mathrm{~s}^{-1}$, indicating that the elongation rate and nucleation rate are highly correlated.

\subsection{Software ActinSimChem}

Although numerous software packages are available for biochemical kinetic modelling, no dedicated, Windows-based software which meets all the needs arising from analysis of any specific actin polymerization system is currently available. Therefore a new standalone software package, ActSimChem, for the here presented Monte Carlo simulation of actin polymerization processes has been developed (for details see Appendix A). ActSimChem provides the possibility for: i) switching between the nSRF and SRF models; ii) input/variation of the model parameters; iii) a compact set of the stochastic modelling algorithms (including the developed modifications of the Gillespie's "direct", the "first reaction", the "next reaction" methods); iv) simulation of actin polymerization (currently up to 21 molecular and 28 chemical actin-related reactions); v) graphical representation of simulation results; vi) storage of the results. The program and its manual can be obtained on request or can be downloaded from the website http://actinsim.uni.lu.

\section{Concluding remarks}

We have developed and compared two stochastic simulation models of actin polymerization processes connecting multiple main actin polymerization-related biochemical reactions including spontaneous and enhanced actin nucleation, association/dissociation at filaments barbed and pointed ends, filament branching, fragmentation and annealing. Additionally the action of different actin-accessory protein that regulate filament dynamics, the structural composition of filaments and the distribution of filament lengths can be simulated. We evaluated the computational efficiency and simulation accuracy of the nSRF and SRF models, that differ in presentation of structural, i.e. ATP-hydrolysis-related, properties of actin filaments. In the majority of numerical tests, the nSRF model considerably saved computational time and still gave outcome characteristics at the same confidence level as the complete SRF model. This result realistically enables the nSRF model to be applied for experimental data fitting and for deriving reaction rate constants on the same manner as reported in [48-50]. For situations were ATP-actin monomers are limiting the SRF model needs, however, to be used.

The presented stochastic methodology forms a considerable improvement upon recently reported models $[23,24]$ in at least four ways. First, the number of already incorporated actin-polymerization reactions is very high. This allows evaluating outcome of complex biosystems closer to physiological situations, thus adding predictive power. Second, the Monte Carlo-based simulation algorithms are efficient and robust. Third, the open-architecture principle in the integrated modelling of actin-associated reactions events and filaments structures ensures flexibility in combination with a broad applicability. This architecture allows upgrading the utilised stochastic simulation algorithm for any newly-developed advanced modelling technique on the one hand, and permits further extension of the simulated system for additional actin-interacting proteins and polymerization mechanisms on the other hand. Fourth, we provide a free and user-friendly software package ActinSimChem, that contains the developed simulation algorithms. The package can be used to simulate in silico the numerical time-resolved outcome under form of the actin filament concentration and distribution of filament lengths for a complex set of actin-polymerization processes. This unique tool for simulation or fitting experimental data, will allow biologists comparing existing actin-polymerization systems and more easily design and interpret complex experiments in which more than "reaction" on actin is taking place.

As demonstrated potential of our model, we performed in this article several numerical experiments on simulations of known and well-established actin polymerization systems. The results of these computer tests underscored important aspects of actin dynamics, namely that: i) under the conditions used, the reactions at pointed ends and by pointed-end capping proteins do not exhibit a significant effect on actin polymerization, unless pointed-end capping proteins work as nucleators; ii) the aging reaction has a minor effect on early state actin polymerization kinetics and only has an effect when 
equilibrium is reached; iii) nucleation and elongation are correlated when considering time evolution of actin in the filamentous form.

\section{Acknowledgments}

This work was supported by Fonds National de la Recherche, Luxembourg (grant nr FNR/05/MA6/17), Human Frontier Science Program (grant nr RGP0058/2005) and the FWO-Vlaanderen to MVT and CA (grant nr G.0157.05), CNRS. AAH was a recipient of an ESF Exchange Visit Grant.

\section{Appendix A. Supplementary data}

Supplementary data associated with this article can be found, in the online version, at doi:10.1016/j.bpc.2008.11.006.

\section{References}

[1] A. Giganti, J. Plastino, B. Janji, M. Van Troys, D. Lentz, C. Ampe, C. Sykes, E. Friederich, Actin-filament cross-linking protein T-plastin increases Arp2/3-mediated actinbased movement, J. Cell Sci. 118 (2005) 1255-1265.

[2] A. Lambrechts, M. Van Troys, C. Ampe, The actin cytoskeleton in normal and pathological cell motility, Int. J. Biochem. Cell Biol. 36 (2004) 1890-1909.

[3] C. Le Clainche, M.F. Carlier, Regulation of actin assembly associated with protrusion and adhesion in cell migration, Physiol. Rev. 88 (2008) 489-513.

[4] M. Van Troys, L. Huyck, S. Leyman, S. Dhaese, J. Vandekerkhove, C. Ampe, Ins and outs of ADF/cofilin activity and regulation, Eur. J. Cell Biol. 87 (2008) 649-667.

[5] D. Yamazaki, S. Kurisu, T. Takenawa, Regulation of cancer cell motility through actin reorganization, Cancer Sci. 96 (2005) 379-386.

[6] A. Giganti, E. Friederich, The actin cytoskeleton as a therapeutic target: state of the art and future directions, Prog. Cell Cycle Res. 5 (2003) 511-525.

[7] F.S. Soo, J.A. Theriot, Large-scale quantitative analysis of sources of variation in the actin polymerization-based movement of Listeria monocytogenes, Biophys. J. 89 (2005) 703-723.

[8] A. Lambrechts, K. Gevaert, P. Cossart, J. Vandekerckhove, M. Van Troys, Listeria comet tails: the actin-based motility machinery at work, Trends Cell Biol. 18 (2008) 220-227.

[9] T.D. Pollard, G.G. Borisy, Cellular motility driven by assembly and disassembly of actin filaments, Cell 112 (2003) 453-465.

[10] A. Mogilner, Mathematics of cell motility: have we got its number? J. Math. Biol. 58 (2009) 105-134.

[11] S. Romero, C. Le Clainche, D. Didry, C. Egile, D. Pantaloni, M.F. Carlier, Formin is a processive motor that requires profilin to accelerate actin assembly and associated ATP hydrolysis, Cell 119 (2004) 419-429.

[12] D.R. Kovar, Molecular details of formin-mediated actin assembly, Curr. Opin. Cell Biol. 18 (2006) 11-17.

[13] M. Pring, M. Evangelista, C. Boone, C. Yang, S.H. Zigmond, Mechanism of formininduced nucleation of actin filaments, Biochemistry 42 (2003) 486-496.

[14] A.E. Carlsson, M.A. Wear, J.A. Cooper, End versus side branching by Arp2/3 complex, Biophys. J. 86 (2004) 1074-1081.

[15] T.D. Pollard, C.C. Beltzner, Structure and function of the Arp2/3 complex, Curr. Opin. Struct. Biol. 12 (2002) 768-774.

[16] R.E. Mahaffy, T.D. Pollard, Kinetics of the formation and dissociation of actin filament branches mediated by Arp2/3 complex, Biophys. J. 91 (2006) 3519-3528.

[17] J.A. Cooper, T.D. Pollard, Effect of capping protein on the kinetics of actin polymerization, Biochemistry 24 (1985) 793-799.

[18] A. Matzavinos, H.G. Othmer, A stochastic analysis of actin polymerization in the presence of twinfilin and gesolin, J. Theor. Biol. (2007).

[19] V. DesMarais, M. Ghosh, R. Eddy, J. Condeelis, Cofilin takes the lead, J. Cell Sci. 118 (2005) 19-26

[20] A.E. Carlsson, Stimulation of actin polymerization by filament severing, Biophys. J. 90 (2006) 413-422.

[21] J.L. McGrath, E.A. Osborn, Y.S. Tardy, C.F. Dewey Jr., J.H. Hartwig, Regulation of the actin cycle in vivo by actin filament severing, Proc. Natl. Acad. Sci. U. S. A. 97 (2000) 6532-6537.

[22] K. Binder, M.H. Kalos, Monte Carlo methods in statistical physics, Springer, Berlin, 1979.

[23] F.J. Brooks, A.E. Carlsson, Actin polymerization overshoots and ATP hydrolysis as assayed by pyrene fluorescence, Biophys. J. 95 (2008) 1050-1062.

[24] J. Roland, J. Berro, A. Michelot, L. Blanchoin, J.L. Martiel, Stochastic severing of actin filaments by actin depolymerizing factor/cofilin controls the emergence of a steady dynamical regime, Biophys. J. 94 (2008) 2082-2094.

[25] J. Fass, C. Pak, J. Bamburg, A. Mogilner, Stochastic simulation of actin dynamics reveals the role of annealing and fragmentation, J. Theor. Biol. 252 (2008) 173-183.

[26] M.M. Yatskou, H. Donker, E.G. Novikov, R.B.M. Koehorst, A. van Hoek, V.V. Apanasovich, T.J. Schaafsma, Nonisotropic excitation energy transport in orga- nized molecular systems: Monte Carlo simulation-based analysis of time-resolved fluorescence, J. Phys. Chem. A 105 (2001) 9498-9508.

[27] P.V. Nazarov, V.V. Apanasovich, V.M. Lutkovski, M.M. Yatskou, R.B. Koehorst M.A. Hemminga, Artificial neural network modification of simulation-based fitting: application to a protein-lipid system, J. Chem. Inf. Comput. Sci. 44 (2004) 568-574

[28] A.F. Maree, A. Jilkine, A. Dawes, V.A. Grieneisen, L. Edelstein-Keshet, Polarization and movement of keratocytes: a multiscale modelling approach, Bull. Math. Biol. 68 (2006) 1169-1211.

[29] C. Neidl, J. Engel, Exchange of ADP, ATP and 1: N6-ethenoadenosine 5'-triphosphate at G-actin. Equilibrium and kinetics, Eur. J. Biochem. 101 (1979) 163-169.

[30] J.L. McGrath, Y. Tardy, C.F. Dewey Jr., J.J. Meister, J.H. Hartwig, Simultaneous measurements of actin filament turnover, filament fraction, and monomer diffusion in endothelial cells, Biophys. J. 75 (1998) 2070-2078.

[31] J. Lippincott-Schwartz, E. Snapp, A. Kenworthy, Studying proteins dynamics in living cells, Nat. Cell Biol. Rev. 2 (2001) 444-456.

[32] B.L. Sprague, R.L. Pego, D.A. Stavreva, J.G. McNally, Analysis of binding reactions by fluorescence recovery after photobleaching, Biophys. J. 86 (2004) 3473-3495.

[33] K. Braeckmans, K. Remaut, R.E. Vandenbroucke, B. Lucas, S.C. De Smedt, J. Demeester Line FRAP with the confocal laser scanning microscope for diffusion measurements in small regions of 3-D samples, Biophys. J. 92 (2007) 2172-2183.

[34] D.T. Gillespie, A general method for numerically simulating the stochastic time evolution of coupled chemical reactions, J. Comput. Phys. 22 (1976) 403-434.

[35] M.A. Gibson, J. Bruck, Efficient exact stochastic simulation of chemical systems with many species and many channels, J. Phys. Chem. A 104 (2000) 1876-1889.

[36] D.T. Gillespie, Approximate accelerated stochastic simulation of chemically reacting systems, J. Chem. Phys. 115 (2001) 1716-1733.

[37] D.T. Gillespie, Exact stochastic simulation of coupled chemical reactions, J. Phys Chem. 81 (1977) 2340-2361.

[38] S.K. Maciver, A.G. Weeds, Actophorin preferentially binds monomeric ADP-actin over ATP-bound actin: consequences for cell locomotion, FEBS Lett. 347 (1994) 251-256.

[39] J.A. Spudich, S. Watt, The regulation of rabbit skeletal muscle contraction. I Biochemical studies of the interaction of the tropomyosin-troponin complex with actin and the proteolytic fragments of myosin, J. Biol. Chem. 246 (1971) 4866-4871.

[40] T. Kouyama, K. Mihashi, Fluorimetry study of N-(1-pyrenyl)iodoacetamidelabelled F-actin. Local structural change of actin protomer both on polymerization and on binding of heavy meromyosin, Eur. J. Biochem. 114 (1981) 33-38.

[41] M.F. Carlier, D. Pantaloni, E.D. Korn, Evidence for an ATP cap at the ends of actin filaments and its regulation of the F-actin steady state, J. Biol. Chem. 259 (1984) 9983-9986.

[42] L. Blanchoin, T.D. Pollard, Hydrolysis of ATP by polymerized actin depends on the bound divalent cation but not profilin, Biochemistry 41 (2002) 597-602.

[43] M. Bindschadler, E.A. Osborn, C.F. Dewey Jr., J.L. McGrath, A mechanistic model of the actin cycle, Biophys. J. 86 (2004) 2720-2739.

[44] D. Vavylonis, Q. Yang, B. O'Shaughnessy, Actin polymerization kinetics, cap structure, and fluctuations, Proc. Natl. Acad. Sci. U. S. A. 102 (2005) 8543-8548.

[45] L.S. Tobacman, E.D. Korn, The kinetics of actin nucleation and polymerization, J. Biol. Chem. 258 (1983) 3207-3214.

[46] D. Sept, J. Xu, T.D. Pollard, J.A. McCammon, Annealing accounts for the length of actin filaments formed by spontaneous polymerization, Biophys. J. 77 (1999) 2911-2919.

[47] A.E. Carlsson, The effect of branching on the critical concentration and average filament length of actin, Biophys. J. 89 (2005) 130-140.

[48] M.M. Yatskou, M. Meyer, S. Huber, M. Pfenniger, G. Calzaferri, Electronic excitation energy migration in a photonic dye-zeolite antenna, ChemPhysChem 4 (2003) 567-587.

[49] P.V. Nazarov, R.B. Koehorst, W.L. Vos, V.V. Apanasovich, M.A. Hemminga, FRET study of membrane proteins: simulation-based fitting for analysis of membrane protein embedment and association, Biophys. J. 91 (2006) 454-466.

[50] D. Marushchak, S. Grenklo, T. Johansson, R. Karlsson, L.B. Johansson, Fluorescence depolarisation studies of filamentous actin analysed with a genetic algorithm, Biophys. J. 93 (2007) 3291-3299.

[51] S. Samarin, S. Romero, C. Kocks, D. Didry, D. Pantaloni, M.F. Carlier, How VASP enhances actin-based motility, J. Cell Biol. 163 (2003) 131-142.

[52] D. Sept, J.A. McCammon, Thermodynamics and kinetics of actin filament nucleation, Biophys. J. 81 (2001) 667-674

[53] T.D. Pollard, Rate constants for the reactions of ATP- and ADP-actin with the ends of actin filaments, J. Cell Biol. 103 (1986) 2747-2754.

[54] D. Schafer, P. Jennings, J. Cooper, Dynamics of capping protein and actin assembly in vitro: uncapping barbed ends by polyphosphoinisitides, J. Cell Biol. 135 (1996) 169-179.

[55] J.R. Kuhn, T.D. Pollard, Real-time measurements of actin filament polymerization by total internal reflection fluorescence microscopy, Biophys. J. 88 (2005) 1387-1402.

[56] A. Mogilner, L. Edelstein-Keshet, Regulation of actin dynamics in rapidly moving cells: a quantitative analysis, Biophys. J. 83 (2002) 1237-1258. 